\title{
PET Imaging of Tumor PD-L1 Expression with a Highly Specific Nonblocking Single-Domain Antibody
}

\author{
Gaochao Lv*1, Xiaorong Sun*2, Ling Qiu ${ }^{1}$, Yan Sun ${ }^{3}, \mathrm{Ke} \mathrm{Li}^{1}$, Qingzhu Liu ${ }^{1}$, Qi Zhao ${ }^{4}$, Songbing Qin ${ }^{4}$, and Jianguo Lin ${ }^{1}$ \\ ${ }^{1}$ Key Laboratory of Nuclear Medicine of Ministry of Health, Jiangsu Key Laboratory of Molecular Nuclear Medicine, Jiangsu \\ Institute of Nuclear Medicine, Wuxi, China; ${ }^{2}$ Department of Nuclear Medicine, Shandong Cancer Hospital and Institute, Shandong \\ First Medical University and Shandong Academy of Medical Sciences, Jinan, China; ${ }^{3}$ Smart Nuclide Biotech, Suzhou, China; and \\ ${ }^{4}$ Department of Radiation Oncology, First Affiliated Hospital of Soochow University, Suzhou, China
}

\begin{abstract}
Although immunotherapy through programmed death $1 /$ programmed death ligand 1 (PD-1/PD-L1) checkpoint blockade has shown impressive clinical outcomes, not all patients respond to it. Recent studies have demonstrated that the expression level of PDL1 in tumors is one of the factors that correlate with PD-1/PD-L1 checkpoint blockade therapy. Herein, a ${ }^{68} \mathrm{Ga}$-labeled single-domain antibody tracer, ${ }^{68} \mathrm{Ga}-\mathrm{NOTA}-\mathrm{Nb} 109$, was designed and developed for specific and noninvasive imaging of PD-L1 expression in a melanoma-bearing mouse model. Methods: The single-domain antibody $\mathrm{Nb} 109$ was labeled with the radionuclide ${ }^{68} \mathrm{Ga}$ through a NOTA chelator. An in vitro binding assay was performed to assess the affinity and binding epitope of Nb109 to PD-L1. The clinical application value of ${ }^{68} \mathrm{Ga}-\mathrm{NOTA}-\mathrm{Nb} 109$ was evaluated by a stability assay; by biodistribution and pharmacokinetics studies; and by PET imaging, autoradiography, and immunohistochemical staining studies on tumor-bearing models with differences in PD-L1 expression. Results: ${ }^{68} \mathrm{Ga}-\mathrm{NOTA}-\mathrm{Nb} 109$ was obtained with a radiochemical yield of more than $95 \%$ and radiochemical purity of more than $98 \%$ in $10 \mathrm{~min}$. It showed a highly specific affinity for PD-L1, with an equilibrium dissociation constant of $2.9 \times 10^{-9} \mathrm{M}$. A competitive binding assay indicated $\mathrm{Nb} 109$ to have a binding epitope different from that of PD-1 and PD-L1 antibody. All biodistribution, PET imaging, autoradiography, and immunohistochemical staining studies revealed that ${ }^{68} \mathrm{Ga}-\mathrm{NOTA}-\mathrm{Nb} 109$ specifically accumulated in A375hPD-L1 tumor, with a maximum uptake of $5.0 \% \pm 0.35 \%$ injected dose/g at $1 \mathrm{~h}$. Conclusion: ${ }^{68} \mathrm{Ga}-\mathrm{NOTA}-\mathrm{Nb} 109$ holds great potential for noninvasive PET imaging of the PD-L1 status in tumors and for timely evaluation of the effect of immune checkpoint targeting treatment.
\end{abstract}

Key Words: immune checkpoints; PD-L1; PET; nanobody tracer

J Nucl Med 2020; 61:117-122

DOI: 10.2967/jnumed.119.226712

Received Jan. 29, 2019; revision accepted Jun. 18, 2019.

For correspondence or reprints contact the following:

Jianguo Lin, Jiangsu Institute of Nuclear Medicine, 20 Qianrong Rd., Wuxi

214063, China.

E-mail: linjianguo@jsinm.org

Ling Qiu, Jiangsu Institute of Nuclear Medicine, 20 Qianrong Rd., Wuxi

214063, China.

E-mail: qiuling@jsinm.org

Songbing Qin, First Affiliated Hospital of Soochow University, Suzhou, China.

E-mail: qin92244@163.com

${ }^{*}$ Contributed equally to this work.

Published online Jun. 28, 2019.

COPYRIGHT (C 2020 by the Society of Nuclear Medicine and Molecular Imaging.
I mmune checkpoints are inhibitory pathways of the immune system that maintain self-tolerance and prevent autoimmunity (1). One of the checkpoint receptors, programmed cell death protein-1 (PD-1), has been widely studied in the context of clinical cancer immunotherapy, which serves as a negative regulator of Tcells (2). On binding to either of its 2 ligands, PD-L1 or PD-L2, PD-1 will initiate an inhibitory signaling cascade through its intracellular signaling domains, including an immunoreceptor tyrosine-based inhibitory motif and immunoreceptor tyrosine-based switch motif (3). By upregulating the expression level of PD-L1, tumor cells are capable of escaping immune recognition and attack (4). Recently, several immunotherapeutic antibodies that block the PD-1/PD-L1 pathway have shown encouraging results in clinical applications, such as nivolumab, pembrolizumab, and atezolizumab (5). The remarkable results prompted the Food and Drug Administration to accelerate approval of pembrolizumab and nivolumab for the treatment of advanced melanoma in late 2014, and nivolumab was also approved for the treatment of non-small cell lung carcinoma in early 2015 (6).

Unfortunately, although the immunotherapy targeted PD-1/PDL1 pathway shows impressive clinical outcomes, not all patients respond to this type of treatment (7). Several studies have demonstrated that the expression level of PD-L1 in tumors might be one factor that correlates with the PD-1/PD-L1 checkpoint blockade therapy $(8-10)$. Thus, it is essential to analyze PD-L1 expression in patients before treatment to potentially avoid an ineffective cure and improve the success rate of immunotherapy. The traditional method for immunohistochemistry is limited in the analysis of PD-L1 expression in tumors because of the highly dynamic and heterogeneous expression of checkpoint molecules (11). Thus, there is an urgent need for real-time, dynamic, and exact detection of PDL1 with high sensitivity and resolution (12). Recently, PET imaging with radiolabeled anti-PD-L1 antibodies showed important advantages over immunohistochemistry in the detection of PD-L1 expression (13). Besides the operator-independent procedure, both primary tumor and metastases can be evaluated by a single noninvasive whole-body imaging procedure, thereby avoiding sampling errors in the case of PD-L1 expression heterogeneity (14). Moreover, PET imaging also allows a more accurate quantitative assessment of the total amount of PD-L1 expression in an individual patient, which could provide important information for determining the treatment method or the dose of anti-PD-L1 therapy (15-19). However, antibody agents often take many days to clear sufficiently from soft tissues and enable imaging of the target (20). An ideal PET imaging agent for PD-L1 should offer high uptake in PD-L1-positive tumors 
and low background signal in a short time (21). Because of their small size and favorable physicochemical properties, single-domain antibodies have attracted considerable attention and hold great potential in the design of PD-L1-targeting imaging agents (22).

Furthermore, prognostic evaluation of PD-L1 level is also crucial for monitoring therapeutic effects (23). Noteworthy is that the tracer and anti-PD-L1 therapeutic antibodies have different binding sites, which is a popular trait for the prognostic evaluation that could not be affected by the therapeutic antibodies in the course of treatment (24). In the current study, we screened and identified a nonblocking single-domain antibody with high specific affinity for PD-L1, namely Nb109. It was further conjugated with the chelator NOTA and labeled by the radionuclide ${ }^{68} \mathrm{Ga}$ (Fig. 1). ${ }^{68} \mathrm{Ga}-\mathrm{NOTA}-\mathrm{Nb} 109$ showed promising clinical value in imaging of PD-L1 expression in xenograft tumors and holds great potential for guiding immunotherapy.

\section{MATERIALS AND METHODS}

\section{Materials}

All reagents were obtained from Sigma-Aldrich unless otherwise stated. p-SCN-Bn-NOTA was purchased from Macrocyclics. The PDL1 antibody KN035 was kindly provided by Alphamab Co. Ltd. The mass spectra were measured by a high-resolution LTQ-Orbitrap XL mass spectrometer interfaced with a heated electrospray ionization source (Thermo Scientific), and the data were processed using Thermo Biopharma Finder 3.0. ${ }^{68} \mathrm{Ga}$ was obtained from a ${ }^{68} \mathrm{Ga} /{ }^{68} \mathrm{Ge}$ generator (IGG-100; Eckert \& Ziegler AG). High-performance liquid chromatography was performed on a Waters 2998 device with a size-exclusion chromatogram (G3000SWXL; Tosoh).

\section{Production and Purification of Single-Domain Antibody}

Anti-PD-L1 single-domain antibody DNA fragments were recloned in a suitable mammalian expression vector, pcDNA4 (catalog number V86220; Invitrogen). Human embryonic kidney suspension-adapted cells, HEK293F, were cultured according to standard protocols. Starter cultures $(30-100 \mathrm{~mL})$ were maintained in $250-\mathrm{mL}$ conical cell culture flasks. To obtain transient cotransfection HEK293F cells, freestyle HEK293F cells were adapted to grow in suspension cultures, reaching high densities using serum-free medium. Then, the cells were transiently transfected using a branched version of polyethyleneimine. Intracellular proteins were harvested by centrifuging cells at $3,000 \mathrm{~g}$ for $5 \mathrm{~min}$. Single-domain antibodies were further purified using immobilized affinity chromatography and ion-exchange liquid chromatography on

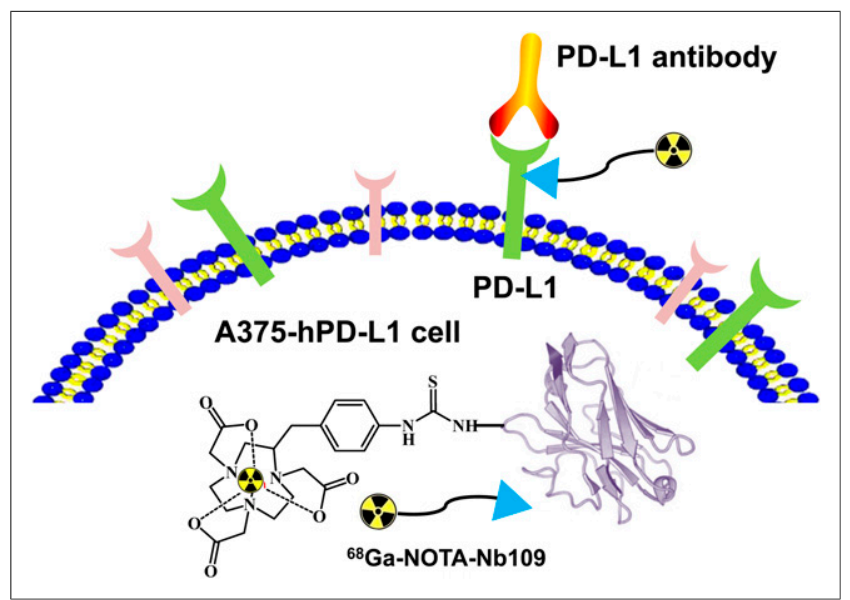

FIGURE 1. Schematic illustration of ${ }^{68}$ Ga-NOTA-Nb109 binding with PD-L1. sulphopropyl resin (GE Healthcare), followed by buffer exchange to phosphate-buffered saline.

\section{Synthesis of ${ }^{69} \mathrm{Ga}-\mathrm{NOTA}-\mathrm{Nb} 109$}

The precursor NOTA-Nb109 was obtained by conjugation of p-SCNBn-NOTA with amino groups of $\mathrm{Nb} 109$ according to a previous report (11). To a solution of $\mathrm{Ga}\left(\mathrm{NO}_{3}\right)_{3}(2.0 \mathrm{nmol})$ in $500 \mu \mathrm{L}$ of $0.25 \mathrm{M}$ sodium acetate, $0.05 \mathrm{M} \mathrm{HCl}$ was added to adjust the $\mathrm{pH}$ of the reaction system to 4.0 , followed by the addition of NOTA-Nb109 $(100 \mu \mathrm{g})$. The mixture was then incubated at room temperature for $10 \mathrm{~min}$ and purified with a PD-10 column.

\section{Synthesis of the Probe ${ }^{68} \mathrm{Ga}-\mathrm{NOTA}-\mathrm{Nb} 109$}

The radionuclide ${ }^{68} \mathrm{Ga}$ was eluted from a ${ }^{68} \mathrm{Ga} /{ }^{68} \mathrm{Ge}$ generator using $0.05 \mathrm{M} \mathrm{HCl}(5 \mathrm{~mL})$ as the fractionated eluent. The single-domain antibody Nb109 $(100 \mu \mathrm{g})$ was mixed with the metallic cation ${ }^{68} \mathrm{Ga}^{3+}(1 \mathrm{~mL})$ and sodium acetate $(0.25 \mathrm{M}, 225 \mu \mathrm{L})$. The reaction mixture was incubated at room temperature for $10 \mathrm{~min}$ and purified by a PD-10 column with saline as the eluent.

The purity and stability of ${ }^{68}$ Ga-NOTA-Nb109 were measured by radio-high-performance liquid chromatography/size-exclusion chromatography using $0.01 \mathrm{M}$ phosphate buffer ( $\mathrm{pH} 7.4)$ as the mobile phase at a flow rate of $1 \mathrm{~mL} / \mathrm{min}$.

\section{Binding Affinity Assay}

The affinity of single-domain antibody $\mathrm{Nb109}$ for immobilized human PD-L1 protein was tested using surface plasmon resonance. All measurements were performed on a Biacore T200 device at $25^{\circ} \mathrm{C}$ using 4-(2-hydroxyethyl)-1-piperazineethanesulfonic acid-buffered saline (0.01 M, pH 7.4; $0.15 \mathrm{M} \mathrm{NaCl} ; 3 \mathrm{mM}$ ethylenediaminetetraacetic acid; $0.005 \%$ polysorbate 20 ) as the running buffer. Briefly, 6 different dilutions of $\mathrm{Nb} 109(0.94,1.85,3.75,7.5,15$, and $30 \mathrm{nM})$ were run at $50 \mu \mathrm{L} / \mathrm{min}$ on a CM5 sensor chip with a high density of human PDL1 protein, and the specific binding signal (response units) was recorded. Nb109 dilutions were allowed to bind with the target protein for $300 \mathrm{~s}$, and dissociation was monitored for $180 \mathrm{~s}$. The equilibrium dissociation constant, $\mathrm{K}_{\mathrm{D}}$, was calculated by fitting the obtained sensor grams to theoretic curves using Biacore Evaluation software.

The competition binding assay was performed by enzyme-linked immunosorbent assay (ELISA). PD-L1-muFc and PD-1-Fc were expressed by HEK293 cell lines (pcDNA4, catalog number V86220; Invitrogen). PD-L1-muFc was coated on the plate as a capture reagent using $0.5 \mu \mathrm{g}$ per well. The plate was incubated at $4^{\circ} \mathrm{C}$ overnight, and the excess of uncoated fusion protein was removed by washing the plate 3 times with phosphate buffer containing $0.01 \%$ polysorbate 20 . Subsequently, $10 \mu \mathrm{g}$ of PD-1-Fc were added, followed by the addition of $\mathrm{Nb} 109$ with a geometric dilution at an initial concentration of $100 \mu \mathrm{g} /$ $\mathrm{mL}$. After incubation at room temperature for $1 \mathrm{~h}, 100 \mu \mathrm{L}$ of anti-His horseradish peroxidase (Abcam) were added to the plate and then reacted for another $1 \mathrm{~h}$. The ELISA was developed by adding $100 \mu \mathrm{L}$ of 3,3',5,5'tetramethylbenzine substrate and subsequent incubation for $25 \mathrm{~min}$ at room temperature. The plates were read at an optical density of $405 \mathrm{~nm}$ with a VersaMax ELISA microplate reader (Molecular Devices).

\section{Cell Lines and Culture Conditions}

Human melanoma cell line A375 and human PD-L1 gene-transfected A375 cells (A375-hPD-L1) were kindly provided by Smart Nuclide Biotech and cultured in Dulbecco modified Eagle medium with $10 \%$ fetal bovine serum (Gibco), penicillin $(100 \mathrm{U} / \mathrm{mL})$, and streptomycin $(0.1 \mathrm{mg} / \mathrm{mL})$. Human breast cancer cells, MCF-7, were obtained from American Type Culture Collection. MCF-7 cells were cultured in Eagle minimum essential medium with $10 \%$ fetal bovine serum and human recombinant insulin $(0.01 \mathrm{mg} / \mathrm{mL}$; Sigma). All cells were grown in a monolayer in Falcon tissue culture dishes (Becton Dickinson) and incubated at $37^{\circ} \mathrm{C}$ in a humidified incubator with $5 \% \mathrm{CO}_{2}$. 


\section{Flow Cytometry for PD-L1 Expression}

The expression level of PD-L1 in A375-hPD-L1 and MCF-7 cell lines was analyzed using a PD-L1 monoclonal antibody (clone 44716) conjugated to phycoerythrin. Flow cytometric analyses were performed on a Beckman Coulter Cytomics FC 500 MPL.

\section{Cellular Uptake and Immunoreactivity}

Cellular uptake was studied according to a previous report (10). The immunoreactive fraction was obtained by the Lindmo assay (25). For a competitive binding assay, the tumor cell (A375-hPD-L1) uptake of ${ }^{68} \mathrm{Ga}-\mathrm{NOTA}-\mathrm{Nb} 109$ was studied with and without pretreatment with a 1,000-fold molar excess of unlabeled Nb109 or KN035. The PD-L1 antibody KN035 has been approved as an investigational new drug for the treatment of advanced or metastatic solid tumors (NCT02827968, NCT03101488, and NCT03248843) $(4,26)$.

\section{Animal Studies}

At 5-7 wk of age, female BALB/c nude mice were inoculated subcutaneously with $1 \times 10^{6}$ A375-hPD-L1 or MCF-7 cells. To further demonstrate the PD-L1-targeting ability of ${ }^{68} \mathrm{Ga}-\mathrm{NOTA}-\mathrm{Nb} 109$ and eliminate individual differences, different cancer cells-A375-hPD-L1, the mixture of A375-hPD-L1/A375 (1/1, v/v), and A375-were simultaneously inoculated into the left hind leg, right hind leg, and right foreleg of the same mouse subcutaneously, respectively. Experiments started when tumors reached a size of approximately $250-350 \mathrm{~mm}^{3}$. All experiments for animal research were conducted in accordance with the principles laid out by the ethical committee of Jiangsu Institute of Nuclear Medicine.

\section{PET Imaging Studies}

PET imaging was performed on an Inveon small-animal PET scanner (Siemens Medical Solutions). Xenografted nude mice were injected with $4.0-5.0 \mathrm{MBq}$ of ${ }^{68} \mathrm{Ga}-\mathrm{NOTA}-\mathrm{Nb} 109$ via the tail vein. For the blocking group, the mice were pretreated with the antibody KN035 $(5 \mathrm{mg} / \mathrm{kg}) 1 \mathrm{~d}$ in advance. The mice were anesthetized with $1.5 \%-2 \%$ isoflurane in a $0.5 \mathrm{~L} / \mathrm{min}$ flow of oxygen. Static imaging (10 min) was performed at 1,2, and $4 \mathrm{~h}$ after injection. Dynamic images were collected for $2 \mathrm{~h}$. The obtained images were reconstructed using 3-dimensional ordered-subset expectation maximization (SP-MAP) without attenuation correction and then processed using a Siemens Inveon Research Workplace (IRW2.0.0.1050). Regions of interest were drawn over tumors and main organs, and average signal levels in the regions were measured.

\section{Autoradiography and Immunohistochemical Staining for PD-L1 Expression}

Ninety minutes after injection of the tracer, the animals were processed for autoradiography and immunohistochemical staining as previously reported $(17,27)$.

\section{Biodistribution and Pharmacokinetics Studies}

Mice with subcutaneous A375-hPD-L1 or MCF-7 xenografts were divided into 2 groups ( $n=5$ each) and received an intravenous injection of $3.7 \mathrm{MBq}$ of ${ }^{68} \mathrm{Ga}-\mathrm{NOTA}-\mathrm{Nb} 109$. At 1 and $2 \mathrm{~h}$ after injection, the mice were killed and the main organs were dissected, washed, weighed, and counted by a $\gamma$ counter. Uptake of the radiotracer was expressed as percentage injected dose per gram of tissue $(\% \mathrm{ID} / \mathrm{g})$. For the pharmacokinetics study, blood samples at different time points $(0,5,15,30,60$, and $120 \mathrm{~min}$ ) were collected and counted using a $\gamma$-counter. Statistical analysis was performed using Origin 7.5 (OriginLab).

\section{RESULTS}

\section{Synthesis and Characterization of ${ }^{68} \mathrm{Ga}-\mathrm{NOTA}-\mathrm{Nb} 109$}

The molecular weight of the obtained single-domain antibody, Nb109, was about $14 \mathrm{kDa}$, which was further determined accurately to be 13,612 amu (Supplemental Fig. 1; supplemental materials are available at http://jnm.snmjournals.org). The conjugate NOTA-Nb109 was prepared by coupling p-SCN-Bn-NOTA with the lysine of anti-PD-L1 single-domain antibody Nb109 (Fig. 2 ). Because there were 3 lysine residues in the framework region of Nb109, a mixture of single- and double-NOTA chelator conjugated with single-domain antibody molecule was produced (Supplemental Fig. 2). The success of the generation of the complex, ${ }^{69}$ Ga-NOTA-Nb109, was demonstrated by mass spectra characterization (Supplemental Fig. 3).

${ }^{68} \mathrm{Ga}-\mathrm{NOTA}-\mathrm{Nb} 109$ was produced with a radiochemical yield of more than $95 \%$ and a radiochemical purity of more than $98 \%$ in sodium acetate buffer ( $\mathrm{pH} 4.0$ ), and the molar activity was determined to be $25.17 \pm 3.26 \mathrm{GBq} / \mu \mathrm{mol}$ (Supplemental Fig. $4 \mathrm{~A})$. The in vitro stability of ${ }^{68} \mathrm{Ga}-\mathrm{NOTA}-\mathrm{Nb} 109$ in phosphate buffer and serum was demonstrated by a radiochemical purity of more than $98 \%$ over $4 \mathrm{~h}$ at $75^{\circ} \mathrm{C}$ (Supplemental Figs. $4 \mathrm{~B}$ and $4 \mathrm{C}$ ). The immunoreactive fraction for ${ }^{68} \mathrm{Ga}-\mathrm{NOTA}-\mathrm{Nb} 109$ was determined to be $67.7 \% \pm 4.8 \%$ (Supplemental Fig. 5).

\section{In Vitro Binding Assay of Nb109 to PD-L1}

As shown in Figure 3A, Nb109 exhibited a strong affinity for PD-L1, with a relatively low equilibrium dissociation constant $\left(\mathrm{K}_{\mathrm{D}}=2.9 \times 10^{-9} \mathrm{M}\right)$, a high binding-rate constant $\left(\mathrm{K}_{\mathrm{a}}=1.6 \times\right.$ $\left.10^{5} \mathrm{M}^{-1} \cdot \mathrm{s}^{-1}\right)$, and a low dissociation-rate constant $\left(\mathrm{K}_{\mathrm{d}}=4.9 \times\right.$ $\left.10^{-4} 1 / \mathrm{s}\right)$. The typical S-type binding curves of $\mathrm{Nb} 109$ and ${ }^{69} \mathrm{Ga}-$ NOTA-Nb109 to PD-L1 by ELISA also indicated their strong binding ability to PD-L1 (Supplemental Fig. 6).

A competitive binding assay showed that the presence of PD-1 does not influence binding of Nb109 to PD-L1, since the typical Stype binding curve between Nb109 and PD-L1 was observed (Fig. 3B). In addition, the existence of $\mathrm{Nb} 109$ did not affect the binding of PD-1 to PD-L1, as the binding curve between PD-1 and PD-L1 showed virtually no change. To confirm the results, we investigated whether KN035 competed with ${ }^{68} \mathrm{Ga}-\mathrm{NOTA}-\mathrm{Nb} 109$ in binding to A375-hPD-L1 cells. After pretreatment with a 1,000-fold excess of KN035 for $30 \mathrm{~min}$, cellular uptake of ${ }^{68}$ Ga-NOTA-Nb109 at $4 \mathrm{~h}$ was comparable to that in the untreated group, but it significantly decreased from $7.17 \% \pm 1.01 \%$ to $1.94 \% \pm 0.53 \%$ by pretreatment

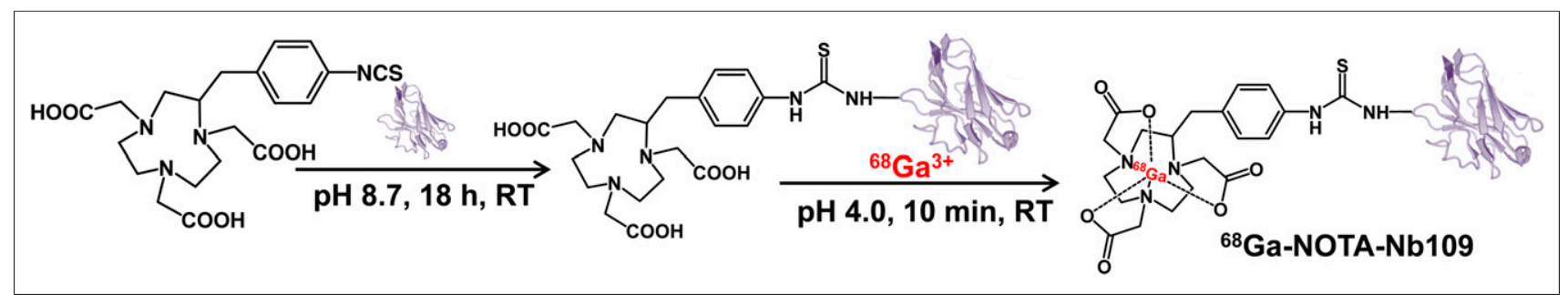

FIGURE 2. Synthetic scheme for ${ }^{68} \mathrm{Ga}-\mathrm{NOTA}-\mathrm{Nb} 109 . \mathrm{RT}=$ room temperature. 


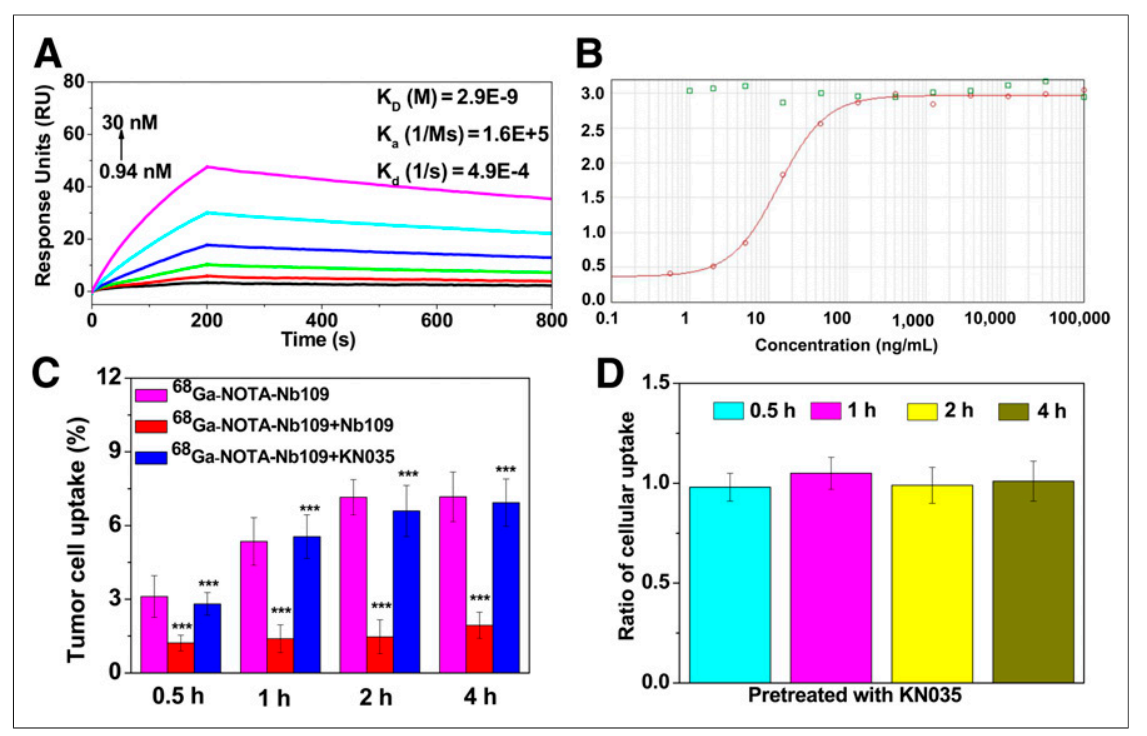

FIGURE 3. In vitro binding studies. (A) Affinity/kinetics surface plasmon resonance study of Nb109 interacting with immobilized recombinant human PD-L1 protein. (B) Binding curves for $\mathrm{Nb} 109$ (red) to PD-L1 at presence of PD-1 (green). (C) Uptake of ${ }^{68} \mathrm{Ga}-\mathrm{NOTA}-\mathrm{Nb} 109$ in A375hPD-L1 with or without pretreatment with 1,000-fold unlabeled Nb109 or KN035 at different times. (D) Ratio of cellular uptake of ${ }^{68} \mathrm{Ga}-\mathrm{NOTA}-\mathrm{Nb} 109$ in A375-hPD-L1 before and after pretreatment with 1,000 -fold KN035 for $0.5,1,2$, and $4 \mathrm{~h} .{ }^{* \star} P<0.001$ vs. ${ }^{68} \mathrm{Ga}-\mathrm{NOTA}-\mathrm{Nb} 109$. $\mathrm{K}_{\mathrm{a}}=$ binding rate constant.

with the same amount of unlabeled Nb109 (Fig. 3C). Furthermore, after pretreatment with 1,000-fold KN035 for 0.5-4 h, cellular uptake of ${ }^{68} \mathrm{Ga}-\mathrm{NOTA}-\mathrm{Nb} 109$ in A375-hPD-L1 was comparable to that in the untreated group, as the ratio of the pretreated group to the untreated group was always close to 1 (Fig. 3D).

Cellular uptake of ${ }^{68} \mathrm{Ga}-\mathrm{NOTA}-\mathrm{Nb} 109$ at $2 \mathrm{~h}$ was $6.3 \% \pm$ $0.31 \%$ and $1.3 \% \pm 0.44 \%$ for A375-hPD-L1 (PD-L1-positive) and MCF-7 (PD-L1-negative) cells, respectively (Supplemental Figs. 7 and 8), indicating the specificity of ${ }^{68}$ Ga-NOTA-Nb109 to PD-L1 in tumor cells.

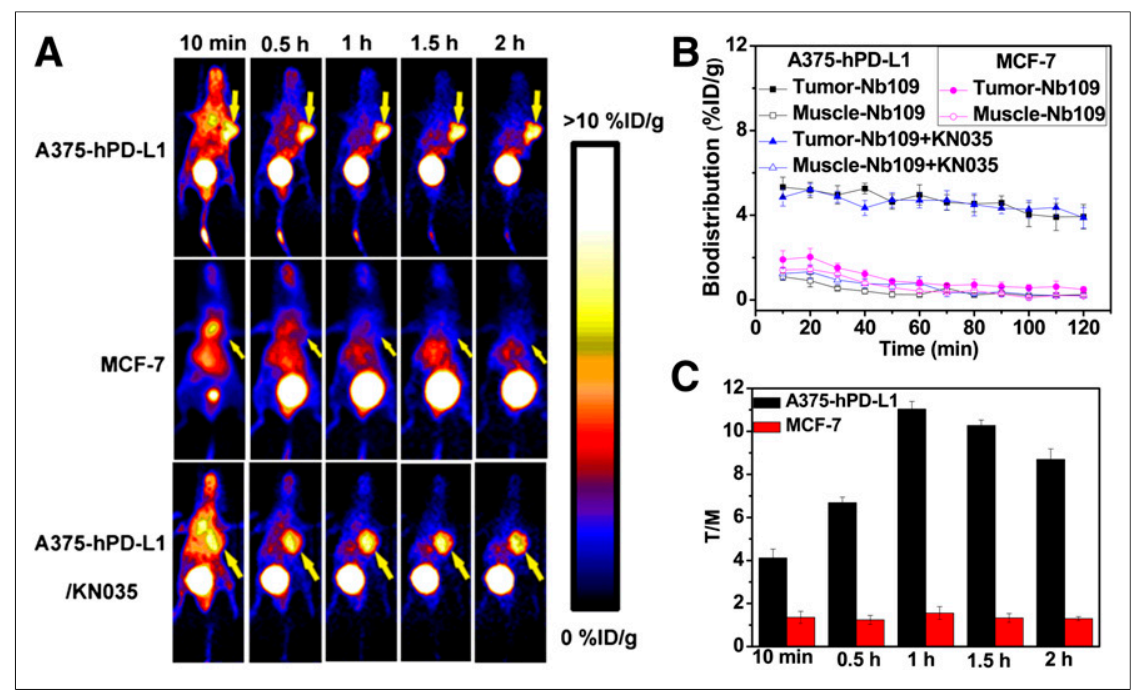

FIGURE 4. PET imaging studies of tumor-bearing models $(n=3)$. (A) Dynamic PET scanning of A375-hPD-L1 (with or without injection of KN035) and MCF-7 tumor-bearing models over 0-2 $\mathrm{h}$ after injection of 4.0-5.0 MBq of ${ }^{68} \mathrm{Ga}-\mathrm{NOTA}-\mathrm{Nb} 109$. (B and C) Biodistribution of ${ }^{68} \mathrm{Ga}-\mathrm{NOTA}-\mathrm{Nb} 109$ and tumor-to-muscle (T/M) ratio analyzed according to quantification analysis of PET images.

\section{PET Imaging}

As shown in Figure 4A, A375-hPD-L1 tumor-bearing mice showed rapid and high uptake by tumors $(5.32 \pm 0.47 \% \mathrm{ID} / \mathrm{g})$ at $10 \mathrm{~min}$ after injection (Fig. 4B). The images were optimal at $1 \mathrm{~h}$ after injection, with the highest tumor-to-muscle ratio being $11.03 \pm 0.36$ (Fig. 4C). However, MCF-7 tumors were not visible at any time during the entire PET acquisition. In the A375-hPDL1 tumor-bearing mice pretreated with the antibody KN035 $(5 \mathrm{mg} / \mathrm{kg})$ for $24 \mathrm{~h}$, tumor uptake of ${ }^{68} \mathrm{Ga}$-NOTA-Nb109 was comparable to that in the animals that were not pretreated with KN035 (Figs. 4A and 4B).

In addition, PET static scanning was performed on 3 types of tumor-bearing mice (Fig. 5). As expected, A375-hPD-L1 tumor was clearly observed and showed the highest uptake of radioactivity at $1 \mathrm{~h}(4.94 \pm$ $0.46 \% \mathrm{ID} / \mathrm{g}$ ) (Figs. 5A and $5 \mathrm{~B}$ ). For the A375-hPD-L1/A375 tumor mixture, uptake was almost half that in A375-hPD-L1 tumor at all time points and was $3.06 \pm 0.31 \%$ $\mathrm{ID} / \mathrm{g}$ at $1 \mathrm{~h}$. PD-L1-negative A375 tumor was not visible at any time during the acquisition. The results correlated well with PD-L1 expression as determined by immunohistochemistry; the PD-L1positive area in A375-hPD-L1 and A375-hPD-L1/A375 tumors was determined to be $30 \% \pm 6.36 \%$ and $15 \% \pm 4.24 \%$, respectively (Fig. $5 \mathrm{C})$. The results were further confirmed by ex vivo autoradiography of tumors after imaging (Fig. 5D).

\section{Biodistribution and Pharmacokinetics Study}

The biodistribution of ${ }^{68} \mathrm{Ga}-\mathrm{NOTA}-\mathrm{Nb} 109$ in A375-hPD-L1 and MCF-7 tumor-bearing mice is presented in Figure 6. At $1 \mathrm{~h}$ after injection, the kidneys showed relatively high uptake (33.66 \pm 3.26 $\% \mathrm{ID} / \mathrm{g})$, whereas the liver $(1.11 \pm 0.41 \%$ $\mathrm{ID} / \mathrm{g})$ and remaining organs $(<1.5 \% \mathrm{ID} / \mathrm{g})$ showed low uptake. Tumor uptake in A375-hPD-L1-bearing mice was rapid and high $(5.0 \pm 0.35 \% \mathrm{ID} / \mathrm{g})$, but that in MCF-7-bearing mice was only $1.7 \pm 0.36$ $\%$ ID/g. At $2 \mathrm{~h}$ after injection, tumor uptake in both A375-hPD-L1- and MCF-7-bearing mice decreased slightly, to $4.05 \pm 0.31$ and $1.46 \pm 0.34 \% \mathrm{ID} / \mathrm{g}$, respectively. The pharmacokinetics study showed that ${ }^{68} \mathrm{Ga}-\mathrm{NOTA}-\mathrm{Nb} 109$ cleared quickly from the blood, with a half-life of $49.79 \mathrm{~min}$ (Supplemental Fig. 9), which resulted in a high tumor-to-muscle ratio (9.33 \pm $0.82)$ and a high tumor-to-blood ratio $(5.48 \pm 0.12)$ at $1 \mathrm{~h}$ after injection in A375-hPD-L1 tumor-bearing mice (Supplemental Fig. 10). Although the tumor-tomuscle ratio decreased slightly to $6.76 \pm$ 0.41 at $2 \mathrm{~h}$ after injection, the tumor-toblood ratio increased to $7.07 \pm 0.11$. In contrast, at $2 \mathrm{~h}$ after injection, both the tumor-to-muscle ratio $(0.98 \pm 0.21)$ and 


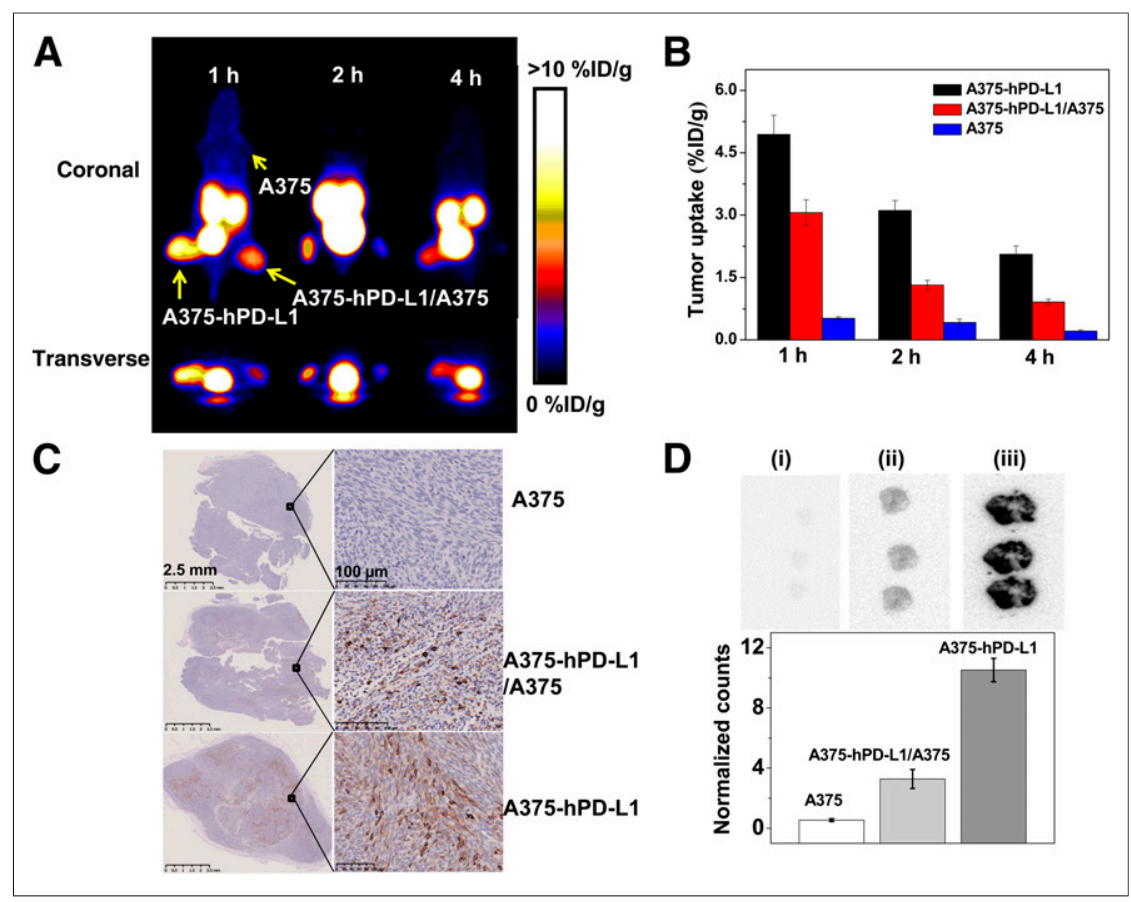

FIGURE 5. PET imaging of tumor-bearing models. (A) Static images at 1,2 , and $4 \mathrm{~h}$ after injection of $2.5 \mathrm{MBq}$ of ${ }^{68} \mathrm{Ga}-\mathrm{NOTA}-\mathrm{Nb} 109(n=3)$. (B) Uptake of ${ }^{68} \mathrm{Ga}-\mathrm{NOTA}-\mathrm{Nb} 109$ in tumors according to quantification analysis of PET images. (C) PD-L1 immunohistochemical staining of tumors ( $n=5$, scale bar $=2.5 \mathrm{~mm}$ in left column and $100 \mu \mathrm{m}$ in right column). (D) Autoradiography analysis of ${ }^{68}$ Ga-NOTA-Nb109 in A375 (i), A375/A375-hPD-L1 (ii), and A375-hPD-L1 (iii) tumor (top) and quantitative analysis of autoradiography (bottom).

the tumor-to-blood ratio $(2.87 \pm 0.13)$ were still low in MCF-7 tumor-bearing mice. In addition, more than $90 \%$ of ${ }^{68} \mathrm{Ga}-\mathrm{NOTA}-$ $\mathrm{Nb} 109$ was intact in the urine at $2 \mathrm{~h}$ after injection, further demonstrating the high in vivo stability of the single-domain antibody (Supplemental Fig. 2).

\section{DISCUSSION}

The immunotherapy-targeted PD-1/PD-L1 pathway shows impressive clinical outcomes, with several radiolabeled PD-L1 imaging agents having been reported recently (13-20). They allow for visualization of PD-L1 status in patients before, during, and after therapeutic intervention and might be able to guide clinical treatment. Recently single-domain antibodies have shown promising results

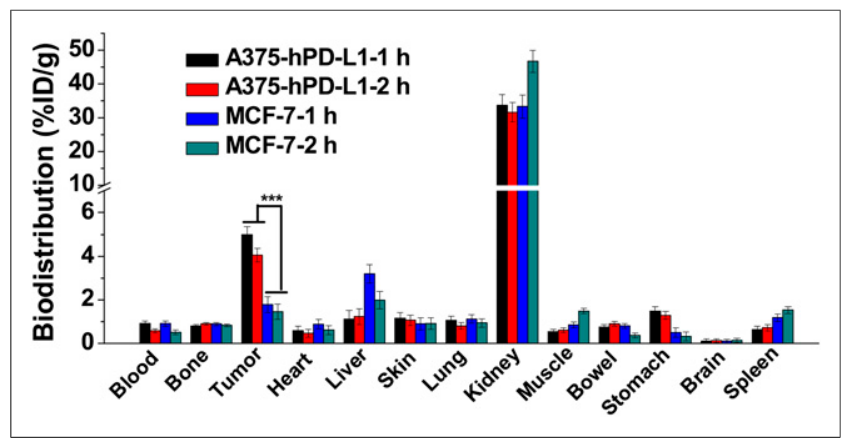

FIGURE 6. Biodistribution analysis at 1 and $2 \mathrm{~h}$ after injection of ${ }^{68} \mathrm{Ga}-$ NOTA-Nb109 in A375-hPD-L1 and MCF-7 tumor-bearing models $(n=$ 5). ${ }^{\star \star *} P<0.001$. as imaging agents because of their favorable physicochemical properties, such as small size, high stability, rapid tumor uptake, and normal-tissue clearance. To facilitate protein isolation, single-domain antibodies are often modified with a histidine tag during protein production, which in turn leads to high kidney uptake and an increasing risk for immunogenicity. These qualities have become obstacles to their clinical application (28). Therefore, we designed a ${ }^{68} \mathrm{Ga}$-labeled single-domain antibody-based probe, ${ }^{68} \mathrm{Ga}$ NOTA-Nb109, without the histidine tag. It could be prepared at high purity and stability, and it holds great potential for PET imaging of PD-L1 expression in cancers.

The binding assay proved the strong binding ability of Nb109 to PD-L1, which had a $\mathrm{K}_{\mathrm{D}}$ value comparable to that of most reported PD-L1 antibodies (12). Furthermore, the similarity between the binding curves for ${ }^{69} \mathrm{Ga}-\mathrm{NOTA}-\mathrm{Nb} 109$ and $\mathrm{Nb} 109$ to PD-L1 demonstrated the strong binding ability of ${ }^{69} \mathrm{Ga}-\mathrm{NOTA}-\mathrm{Nb} 109$ to PD-L1 and indicated that conjugation of the chelator NOTA with ${ }^{68} \mathrm{Ga}^{3+}$ had no effect on the affinity of Nb109 for PD-L1. Six-fold higher uptake of radioactivity in A375-hPD-L1 cells than in MCF-7 cells further confirmed the specificity of ${ }^{68} \mathrm{Ga}-\mathrm{NOTA}-\mathrm{Nb} 109$ for PDL1. All the results demonstrate that ${ }^{68} \mathrm{Ga}$ NOTA-Nb109 binds tumor cells in vitro in a PD-L1 expressiondependent manner.

Compared with most previously reported single-domain antibody-based imaging agents with a histidine tag (29), our tracer, ${ }^{68}$ Ga-NOTA-Nb109, showed much lower kidney retention because of lack of a histidine tag, as can be seen from the PET imaging and biodistribution studies. A higher tumor uptake in A375-hPD-L1bearing mice than in MCF-7-bearing mice was observed, which was attributed to the overexpression of PD-L1 in A375-hPD-L1 tumor. This finding demonstrated that ${ }^{68}$ Ga-NOTA-Nb109 could specifically bind with PD-L1 in vivo. The retention of radioactivity in tumor remained relatively stable for $2 \mathrm{~h}$, but radiotracer cleared quickly from muscle and blood, resulting in a high tumor-to-background ratio in A375-hPD-L1 tumor. Because of the low uptake in MCF-7 tumor, the tumor-to-background ratio was correspondingly low and tumors were not visible during the imaging process.

The competitive binding assay indicated that in vitro binding of Nb109 to PD-L1 was not influenced by PD-1 or KN035, even under a 1,000 -fold excess, but could be blocked by nearly $80 \%$ by the same amount of unlabeled Nb109. This finding demonstrated the difference in binding epitopes between Nb109 and PD-1 or PD-L1 antibody, as well as the specificity of the tracer. For in vivo studies, this finding also confirmed that there was no impact on tumor uptake of ${ }^{68} \mathrm{Ga}-\mathrm{NOTA}-\mathrm{Nb} 109$ before and after injection of KN035, since comparable tumor uptake and images were obtained. Thus, the difference in binding sites between ${ }^{68}$ Ga-NOTA-Nb109 and anti-PD-L1 antibodies to PD-L1 was demonstrated. The nonblocking binding property of ${ }^{68} \mathrm{Ga}-\mathrm{NOTA}-\mathrm{Nb} 109$ endowed it with great potential for detection of PD-L1 and evaluation of prognosis in a 
clinical setting, as it could avoid the effect of anti-PD-L1 antibodies in the course of treatment.

Comparison of PET images of the 3 types of tumor-bearing mice clearly revealed that ${ }^{68} \mathrm{Ga}-\mathrm{NOTA}-\mathrm{Nb} 109$ tended to accumulate in A375-hPD-L1 tumors because of the high PD-L1 expression. In A375-hPD-L1/A375 tumors, the PD-L1 expression decreased because of the doping of A375 cells, and thus the uptake of ${ }^{68} \mathrm{Ga}-$ NOTA-Nb109 decreased as anticipated. Moreover, since A375 cells had a low PD-L1 expression, A375 tumors were not visible during scanning. This finding was further confirmed by immunohistochemistry and autoradiography of the tumors after imaging. All results indicated that ${ }^{68} \mathrm{Ga}-\mathrm{NOTA}-\mathrm{Nb} 109$ could selectively accumulate in PD-L1-positive A375-hPD-L1 tumor and rapidly clear from muscle and blood, resulting in a high tumor-to-background ratio.

\section{CONCLUSION}

A ${ }^{68}$ Ga-labeled single-domain antibody, ${ }^{68}$ Ga-NOTA-Nb109, was developed for PET imaging of PD-L1 in vivo. The tracer has a high affinity toward PD-L1, was generated at high radiochemical yield and radiochemical purity, and showed selective accumulation in PD-L1positive tumors and rapid clearance from muscle and blood, resulting in a high tumor-to-background ratio. All results indicate that ${ }^{68} \mathrm{Ga}$-NOTA$\mathrm{Nb} 109$ has great potential for detecting PD-L1, evaluating therapeutic effect, and optimizing the prescription of PD-1/PD-L1 checkpoint blockade therapy.

\section{DISCLOSURE}

This work was financially supported by the National Natural Science Foundation of China (81972906), the Natural Science Foundation of Jiangsu Province (BK20181128), Project 333 of Jiangsu Province (BRA2016518 and LGY2018086), the Key Youth Medical Talent Project of Jiangsu Province (QNRC2016626 and QNRC2016629), the Science Technology Development Project of Wuxi (WX18IIAN049), and the Precision Medical Project of the Wuxi Commission of Health and Family Planning (J201806). No other potential conflict of interest relevant to this article was reported.

\section{KEY POINTS}

QUESTION: How can PD-L1 expression be detected rapidly and accurately in living subjects?

PERTINENT FINDINGS: A single-domain antibody-based PET tracer, ${ }^{8} \mathrm{Ga}-\mathrm{NOTA}-\mathrm{Nb} 109$, with a high affinity for PD-L1 was developed. PET imaging studies on tumor-bearing models with high, medium, and low PD-L1 expression demonstrated that the tracer could distinctly distinguish different tumors and noninvasively quantify PD-L1 expression in tumors.

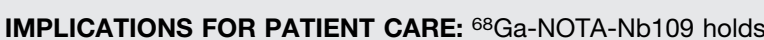
great potential for detecting PD-L1, evaluating the immunotherapeutic effect, and optimizing the prescription of PD-1/PD-L1 checkpoint blockade therapy.

\section{REFERENCES}

1. Francis DM, Thomas SN. Progress and opportunities for enhancing the delivery and efficacy of checkpoint inhibitors for cancer immunotherapy. Adv Drug Deliv Rev. 2017;114:33-42.

2. Ohaegbulam KC, Assal A, Lazar-Molnar E, et al. Human cancer immunotherapy with antibodies to the PD-1 and PD-L1 pathway. Trends Mol Med. 2015;21:24-33.
3. Bellmunt J, Powles T, Vogelzang NJ. A review on the evolution of PD-1/PD-L1 immunotherapy for bladder cancer: the future is now. Cancer Treat Rev. 2017;54:58-67.

4. Zhang F, Wei H, Wang X, et al. Structural basis of a novel PD-L1 nanobody for immune checkpoint blockade. Cell Discov. 2017;3:17004-17016.

5. Emens LA, Ascierto PA, Darcy PK, et al. Cancer immunotherapy: opportunities and challenges in the rapidly evolving clinical landscape. Eur J Cancer. 2017;81:116-129.

6. Zhan MM, Hu X, Liu X, et al. From monoclonal antibodies to small molecules: the development of inhibitors targeting the PD-1/PD-L1 pathway. Drug Discov Today. 2016;21:1027-1036.

7. Geng Q, Jiao P, Jin P, et al. PD-1/PD-L1 inhibitors for immuno-oncology: from antibodies to small molecules. Curr Pharm Des. 2018;23:6033-6041.

8. Hettich M, Braun F, Bartholomä MD, et al. High-resolution PET imaging with therapeutic antibody-based PD-1/PD-L1 checkpoint tracers. Theranostics. 2016;6:1629 1640 .

9. England CG, Ehlerding EB, Hernandez R, et al. Preclinical pharmacokinetics and biodistribution studies of ${ }^{89} \mathrm{Zr}$-labeled pembrolizumab. J Nucl Med. 2017;58: $162-168$.

10. Du Y, Liang X, Li Y, et al. Nuclear and fluorescent labeled PD-1-liposomeDOX- ${ }^{64} \mathrm{Cu} / \mathrm{IRDye} 800 \mathrm{CW}$ allows improved breast tumor targeted imaging and therapy. Mol Pharm. 2017;14:3978-3986.

11. Natarajan A, Mayer AT, Xu L, et al. Novel radiotracer for immunoPET imaging of PD-1 checkpoint expression on tumor infiltrating lymphocytes. Bioconjug Chem. 2015;26:2062-2069.

12. González Trotter DE, Meng X, McQuade P, et al. In vivo imaging of the programmed death ligand 1 by ${ }^{18} \mathrm{~F}$ positron emission tomography. J Nucl Med. 2017;58:1852-1857.

13. Donnelly DJ, Smith RA, Morin P, et al. Synthesis and biological evaluation of a novel ${ }^{18} \mathrm{~F}$-labeled adnectin as a PET radioligand for imaging PD-L1 expression. J Nucl Med. 2018;59:529-535.

14. Mayer AT, Natarajan A, Gordon SR, et al. Practical immuno-PET radiotracer design considerations for human immune checkpoint imaging. J Nucl Med. 2017;58:538-546.

15. Truillet C, Oh HLJ, Yeo SP, et al. Imaging PD-L1 expression with immunoPET. Bioconjug Chem. 2018;29:96-103.

16. Chatterjee S, Lesniak WG, Miller MS, et al. Rapid PD-L1 detection in tumors with PET using a highly specific peptide. Biochem Biophys Res Commun. 2017; 483:258-263.

17. De Silva RA, Kumar D, Lisok A, et al. Peptide-based ${ }^{68} \mathrm{Ga}$-PET radiotracer for imaging PD-L1 expression in cancer. Mol Pharm. 2018;15:3946-3952.

18. Kumar D, Lisok A, Dahmane E, et al. Peptide-based PET quantifies target engagement of PD-L1 therapeutics. J Clin Invest. 2019;129:616-630.

19. Lesniak WG, Chatterjee S, Gabrielson M, et al. PD-L1 detection in tumors using $\left[{ }^{64} \mathrm{Cu}\right]$ atezolizumab with PET. Bioconjug Chem. 2016;27:2103-2110.

20. Natarajan A, Patel CB, Habte F, et al. Dosimetry prediction for clinical translation of ${ }^{64} \mathrm{Cu}$-pembrolizumab immunoPET targeting human PD-1 expression. Sci Rep. 2018;8:633-644.

21. England CG, Jiang D, Ehlerding EB, et al. ${ }^{89} \mathrm{Zr}$-labeled nivolumab for imaging of T-cell infiltration in a humanized murine model of lung cancer. Eur J Nucl Med Mol Imaging. 2018;45:110-120.

22. Schumacher D, Helma J, Schneider AFL, et al. Nanobodies: chemical functionalization strategies and intracellular applications. Angew Chem Int Ed Engl. 2018;57:2314-2333.

23. Wang Q, Lou W, Di W, et al. Prognostic value of tumor PD-L1 expression combined with CD8 + tumor infiltrating lymphocytes in high grade serous ovarian cancer. Int Immunopharmacol. 2017;52:7-14.

24. Bailly C, Cléry P, Faivre-Chauvet A, et al. Immuno-PET for clinical theranostic approaches. Int J Mol Sci. 2016;18:57-69.

25. Lindmo T, Boven E, Cuttitta F, et al. Determination of the immunoreactive fraction of radiolabeled monoclonal antibodies by linear extrapolation to binding at infinite antigen excess. J Immunol Methods. 1984;72:77-89.

26. Li D, Cheng S, Zou S, et al. Immuno-PET imaging of ${ }^{89} \mathrm{Zr}$ labeled anti-PD-L1 domain antibody. Mol Pharm. 2018;15:1674-1681.

27. Zhou Z, Vaidyanathan G, McDougald D, et al. Fluorine-18 labeling of the HER2-targeting single-domain antibody 2Rs15d using a residualizing label and preclinical evaluation. Mol Imaging Biol. 2017;19:867-877.

28. Xavier C, Vaneycken I, D'huyvetter M, et al. Synthesis, preclinical validation, dosimetry, and toxicity of ${ }^{68} \mathrm{Ga}-\mathrm{NOTA}$-anti-HER2 nanobodies for iPET imaging of HER2 receptor expression in cancer. J Nucl Med. 2013;54:776-784.

29. Broos K, Keyaerts M, Lecocq Q, et al. Non-invasive assessment of murine PDL1 levels in syngeneic tumor models by nuclear imaging with nanobody tracers. Oncotarget. 2017;8:41932-41946. 